\title{
Construction of Saccharomyces cerevisiae Strains With Enhanced Ethanol Tolerance by Mutagenesis of the TATA-Binding Protein Gene and Identification of Novel Genes Associated With Ethanol Tolerance
}

\author{
Jungwoo Yang, ${ }^{1}$ Ju Yun Bae, ${ }^{1}$ Young Mi Lee, ${ }^{1}$ Hyeji Kwon, ${ }^{3}$ Hye-Yun Moon, ${ }^{4}$ \\ Hyun Ah Kang, ${ }^{4}$ Su-bog Yee, ${ }^{1}$ Wankee Kim, ${ }^{5}$ Wonja Choi ${ }^{1,2,3}$ \\ ${ }^{I}$ Microbial Resources Research Center, College of Natural Sciences, Ewha Womans, \\ University, Seoul, Korea; telephone: +82-2-3277-2892; fax: +82 23277 2385; \\ e-mail:wjchoi@ewha.ac.kr \\ ${ }^{2}$ Department of Life Science, College of Natural Sciences, Ewha Womans, University, \\ Seoul, Korea \\ ${ }^{3}$ Division of Life and Pharmaceutical Sciences, Ewha Womans University, Seoul, Korea \\ ${ }^{4}$ Department of Life Science, College of Natural Sciences, Chung-Ang University, \\ Seoul, Korea \\ ${ }^{5}$ Institute for Medical Sciences, School of Medicine, Ajou University, Suwon, Korea; \\ telephone: +82-31-219-4506; fax: +82 31219 4508; e-mail: kinesin03@hanmail.net \\ Received 14 October 2010; revision received 28 February 2011; accepted 7 March 2011 \\ Published online 17 March 2011 in Wiley Online Library (wileyonlinelibrary.com). DOI 10.1002/bit.23141
}

\begin{abstract}
Since elevated ethanol is a major stress during ethanol fermentation, yeast strains tolerant to ethanol are highly desirable for the industrial scale ethanol production. A technology called global transcriptional machinery engineering (gTME), which exploits a mutant library of SPT15 encoding the TATA-binding protein of Saccharomyces cerevisiae (Alper et al., 2006; Science 314: 1565-1568), seems to a powerful tool for creating ethanol-tolerant strains. However, the ability of created strains to tolerate high ethanol on rich media remains unproven. In this study, a similar strategy was used to obtain five strains with enhanced ethanol tolerance (ETS1-5) of S. cerevisiae. Comparing global transcriptional profiles of two selected strains ETS2 and ETS3 with that of the control identified 42 genes that were commonly regulated with twofold change. Out of 34 deletion mutants available from a gene knockout library, 18 were ethanol sensitive, suggesting that these genes were closely associated with ethanol tolerance. Eight of them were novel with most being functionally unknown. To establish a basis for future industrial applications, strains iETS2 and iETS3 were created by integrating the SPT15 mutant alleles of ETS2 and ETS3 into the chromosomes, which also exhibited enhanced ethanol tolerance and survi-
\end{abstract}

Correspondence to: W. Choi and W. Kim

Contract grant sponsor: Korea Ministry of Education, Science and Technology Contract grant number: 2009-0081512; 2007-2005047

Additional Supporting Information may be found in the online version of this article. val upon ethanol shock on a rich medium. Fermentation with $20 \%$ glucose for $24 \mathrm{~h}$ in a bioreactor revealed that iETS2 and iETS3 grew better and produced approximately 25\% more ethanol than a control strain. The ethanol yield and productivity were also substantially enhanced: $0.31 \mathrm{~g} / \mathrm{g}$ and $2.6 \mathrm{~g} / \mathrm{L} / \mathrm{h}$, respectively, for control and $0.39 \mathrm{~g} / \mathrm{g}$ and $3.2 \mathrm{~g} / \mathrm{L} / \mathrm{h}$, respectively, for iETS2 and iETS3. Thus, our study demonstrates the utility of gTME in generating strains with enhanced ethanol tolerance that resulted in increase of ethanol production. Strains with enhanced tolerance to other stresses such as heat, fermentation inhibitors, osmotic pressure, and so on, may be further created by using gTME. Biotechnol. Bioeng. 2011;108: 1776-1787.

(C) 2011 Wiley Periodicals, Inc.

KEYWORDS: ethanol stress; SPT15; mutagenesis; ethanol tolerance; DNA microarray

\section{Introduction}

During ethanol production, ethanol-producing microorganisms confront multiple stresses such as high initial substrate concentration, increased ethanol concentration, and accumulation of toxic byproducts. In addition to rapid growth and efficient fermentation capacity, the ability to tolerate these stresses is an important factor in choosing an 
ethanol producer (Ding et al., 2009; Gibson et al., 2007; Zhao and Bai, 2009). Saccharomyces cerevisiae has been used as a primary microorganism for ethanol production on an industrial scale. The accumulation of ethanol to toxic concentrations during fermentation is the major stress that causes reduced ethanol production and eventual stuck fermentations (Gibson et al., 2007). Thus, the development of $S$. cerevisiae strains that can endure ethanol stress is both prudent and important. One way to address such an issue is to understand the mechanisms underlying ethanol stress tolerance. For this, two different molecular approaches have recently been used to identify genes involved in ethanol tolerance: genome-wide DNA microarray analysis (Alexandre et al., 2001; Chandler et al., 2004; Dinh et al., 2009; Hirasawa et al., 2007; Marks et al., 2008; Rossignol et al., 2003; Varela et al., 2005; Wu et al., 2006) and screening of single gene knockout (SGKO) libraries (Fujita et al., 2006; Kubota et al., 2004; Yazawa et al., 2007; Teixeira et al., 2009; van Voorst et al., 2006; Yoshikawa et al., 2009). However, the issue with these two approaches is that a huge number of target genes have been identified, representing as much as $5-10 \%$ of genes encoded in the yeast genome and that few successful examples have been documented (Hirasawa et al., 2007; Yazawa et al., 2007).

An alternative approach to develop ethanol-tolerant strains (ETSs) is global transcriptional machinery engineering (gTME). This approach was first used to create a strain with enhanced ethanol tolerance by generating mutations of TATA-binding protein encoded by SPT15, which could grow at a formerly lethal ethanol concentration (Alper et al., 2006). However, other authors reported that this enhanced ethanol tolerance was not reproduced on a rich medium (Baerends et al., 2009), which is not optional for industrial applications.

Nevertheless, SPT15 mutations altered the transcription profile (Alper et al., 2006). In addition, SPT15 mutations were pleiotropic (Eisenmann et al., 1989) and some mutations in the regulatory domain of SPT15 resulted in transcriptional increase (Cang et al., 1999). These observations indicate that different mutations of SPT15 may induce expression of different sets of genes. It was of our concern whether point mutations different those introduced by Alper et al. (2006) could enhance ethanol tolerance on a rich medium. In this study, five ETSs containing different SPT15 mutant alleles were obtained and the effect of SPT15 mutations on ethanol production was examined, re-empathizing the usefulness of gTME as a tool for creating strains tolerant to ethanol and hopefully to other various stresses.

\section{Materials and Methods}

\section{Yeast Strains and Media}

S. cerevisiae L3262 (MAT- $\alpha$; ura3-52 leu2-3,112 his4-34) and BY4741 (MATa his3 11 leu2 $\Delta 0$ met15 $\Delta 0$ ura3 $\Delta 0$ ) were used as transformation recipients. The non-essential haploid $S$. cerevisiae deletion library was used for the verification of identified genes. Unless otherwise mentioned, yeast cells were grown at $30^{\circ} \mathrm{C}$ in $\mathrm{YPD}(1 \%$ yeast extract, $2 \%$ peptone, $2 \%$ glucose, and $1.5 \%$ agar for solid plates) for non-selective propagation or yeast synthetic complete (YSCD) medium ( $0.67 \%$ yeast nitrogen base without amino acids, amino acid supplement mixture, $2 \%$ dextrose, and 1.5\% noble agar for solid plates) for selective propagation.

\section{Molecular Methods}

Plasmid preparation, cloning, and sequencing were performed as previously described (Sambrook and Russell, 2001). Escherichia coli strain DH5 $\alpha$ was used as a host for plasmid preparation.

\section{Reverse Transcription-Polymerase Chain Reaction (RT-PCR) and PCR}

For RT, total RNA was prepared from exponentially growing cells. First strand cDNAs were synthesized by transcribing $2 \mu \mathrm{g}$ of total RNAs with random hexamers and $200 \mathrm{U}$ of $\mathrm{M}-\mathrm{MuLV}$ reverse transcriptase (Promega, Madison, WI) as recommended by the manufacturer. Oligonucleotides used for PCR are listed in Supplementary Table I. The amplification conditions were $95^{\circ} \mathrm{C}$ for $1 \mathrm{~min}, 55-60^{\circ} \mathrm{C}$ for $1 \mathrm{~min}$, and $72^{\circ} \mathrm{C}$ for the appropriate period of time depending on the length of DNA to be amplified for 20 cycles for RT-PCR and 30 cycles for regular PCR. If necessary, PCR products were purified by gel elution, cloned into the pGEM-T easy vector (Promega), and sequenced.

\section{SPT15 Mutant Library Construction}

The open reading frame (ORF) of SPT15wt was PCRamplified from genomic DNA as a template with primers SPT15ORF-S and SPT15ORF-AS and cloned into the pGEMT-easy vector (Promega), yielding pT-SPT15. The SPT15 mutant library was generated by using the GeneMorph II random mutagenesis kit (Stratagene, La Jolla, CA) with pT-SPT15 as template and using the aforementioned primers. PCR products were digested with BamHI and EcoRI, and cloned into a pRS316-derived plasmid, pRS316-GCYH2gR, in which cloned genes are placed under control of glyceraldehyde-3-phosphate dehydrogenase promoter $\left(T D H 3_{\mathrm{P}}\right)$ and galactose-1-phosphate uridyl transferase terminator $\left(G A L 7_{\mathrm{T}}\right)$. The resulting plasmids were transformed into $E$. coli $\mathrm{DH} 5 \alpha$ and incubated at $30^{\circ} \mathrm{C}$ (to prevent outgrowth of fast-growing cells) to generate a primary library for SPT15 mutants with total colony number being $4 \times 10^{6}$. From the sequencing of 20 randomly selected colonies, the molecule-based mutation rate was determined to be $70 \%$. Mutations were found at more than one site, mostly 3-5, in 14 colonies, with the remainder being the wild type. One of these wild-type 
plasmids was used as the control vector (pSPT15wt). Following amplification and large-scale preparation, the library plasmids $(500 \mu \mathrm{g})$ were transformed into S. cerevisiae L3262 and incubated at $25^{\circ} \mathrm{C}$ (to prevent outgrowth of fastgrowing cells) on solid YSCD-Ura. The total number of yeast colonies was approximately $5 \times 10^{6}$ with a transformation efficiency of approximately $1 \times 10^{4}$ colony forming units $(\mathrm{CFU}) / \mu \mathrm{g}$ DNA. All the colonies were harvested by scrubbing the surfaces of plates with $15 \mathrm{~mL}$ YSCD-Ura to prepare a yeast library for SPT15 mutants. After fourfold propagation in cell number at $25^{\circ} \mathrm{C}$, aliquots of the cell suspension were stored at $-80^{\circ} \mathrm{C}$ in the presence of $20 \%$ glycerol until used.

\section{Yeast Transformation}

All plasmids for yeast transformation were manually prepared without RNA digestion. The DNA concentration was roughly measured by comparing the band intensity with that of control DNA of known concentration. This mixture of DNAs and RNAs was used for yeast transformation as previously described (Gietz and Woods, 2002).

\section{Spot Assay and Ethanol Susceptibility Assay}

For spot assay, aliquots $(5 \mu \mathrm{l})$ of cells grown to an optical density at $600 \mathrm{~nm}\left(\mathrm{OD}_{600}\right)$ of 1.0 were tenfold serially diluted and spotted onto solid synthetic or rich media containing appropriate concentrations of ethanol. Plates were incubated at $30^{\circ} \mathrm{C}$ for $4-6$ days.

For ethanol susceptibility assay, Cells grown to $\mathrm{OD}_{600}$ of 1.0 were harvested, equally divided into fresh YSCD-Ura media containing $12.5 \%$ and $15 \%$ ethanol $(\mathrm{v} / \mathrm{v})$, and incubated at $30^{\circ} \mathrm{C}$ for $4-6 \mathrm{~h}$. At appropriate time points, aliquots were properly diluted and plated onto solid YPD. Cell viability was measured as a function of time and expressed as the relative number of CFU.

\section{Transcriptome Profiling and Data Analysis}

S. cerevisiae $30 \mathrm{~K}$ oligo microarrays (MYcroarray, Ann Arbor, MI) were used for transcriptome profiling. Total RNA was prepared from exponentially growing cells and RNA quality control for microarray analysis were performed as described previously (Park et al., 2007). cDNAs incorporated with aminoallyl-dUTP were synthesized from 40 to $50 \mu \mathrm{g}$ of total RNA using an Aminoallyl post DNA Labeling kit (GeneChem, Daejeon, Korea) and a superscript reverse transcriptase (Invitrogen, Carlsbad, CA). The synthesized cDNA was labeled with NHS-ester Cy dyes and used for hybridization. Hybridized slides were washed by SSC buffer, and then scanned with a ScanArray 5000 scanner (HewlettPackard, Palo Alto, CA). Raw microarray data were analyzed by using ArrayNorm (http://genome.tugraz.at/), a platformindependent Java tool for normalization and statistical analysis (Pieler et al., 2004). Clustering for genes with the average change higher than twofold was carried out using Cluster 3.0 (http://rana.lbl.gov/EisenSoftware.htm). Enrichment of functional categories among differentially expressed genes was analyzed using the MIPS Functional Catalogue (http://mips.gsf.de). Specific gene functions were based on the Saccharomyces Genome Database (http:// www.yeastgenome.org), and transcription factor-binding sties were analyzed by YEASTRACT (http://www.yeastract. com/index.php). To validate DNA microarray data, semiquantitative reverse transcription PCR was performed as described previously (Oh et al., 2004) with the RNA samples used for microarray experiments.

\section{Genomic Integration}

The DNA fragments covering the TDH3p, ORF, and GAL3T were excised from pSPT15wt, pSPT15-M2, and pSPT15-M3 and cloned into the integrating vector pRS406. The resulting plasmids were linearized with $A p a I$ and transformed into $S$. cerevisiae L3262. Genomic integration was verified by PCR with primer sets shown in Supplementary Table 1.

\section{Fermentation}

Ethanolic fermentations were performed at $30^{\circ} \mathrm{C}$ with cells initially adjusted to an $\mathrm{OD}_{600}$ of $\sim 1.0$ in $500 \mathrm{~mL}$ YPD20 (2\% peptone, $1 \%$ yeast extract, $0.02 \%\left(\mathrm{NH}_{4}\right)_{2} \mathrm{SO}_{4}$, and $20 \%$ glucose) in 1-L bioreactor with $\mathrm{pH}$ maintained at 5.5 and oxygen supplied at $200 \mathrm{~mL} / \mathrm{min}$. The cultures were agitated at $400 \mathrm{rpm}$ with antifoaming agent added. Samples were collected at appropriate time points and analyzed for concentration of glucose, ethanol, and glycerol produced during fermentation by performing high-pressure liquid chromatography (HPLC). The samples were loaded onto Aminex HPX$87 \mathrm{H}$ column (Bio-Rad, Hercules, CA) set to $60^{\circ} \mathrm{C}$ and eluted with $0.5 \mathrm{mM} \mathrm{H}_{2} \mathrm{SO}_{4}$ at a constant flow rate of $0.6 \mathrm{~mL} / \mathrm{min}$. Peaks were detected by a refractive index detector and quantified according to a calibration curve for each of glucose, ethanol, and glycerol molecules. Cell growth was monitored by measuring the $\mathrm{OD}_{600}$ with appropriate dilutions.

\section{Results}

\section{Identification of Ethanol-Tolerant Strains}

To obtain ETSs by screening of a yeast SPT15 mutant library constructed in the present study, $5 \times 10^{6}$ colony forming units were spread on the solid YSCD-Ura medium supplemented with $12.5 \%$ ethanol and incubated at $30^{\circ} \mathrm{C}$. Seven days after, 15 colonies had developed in the presence of $12.5 \%$ ethanol. The ethanol tolerance of the 15 colonies was examined by a spot assay on the solid YSCD-Ura medium containing up to $15 \%$ ethanol. As a result, five ETSs (ETS1-5) were obtained. All five strains tolerated 15\% ethanol, whereas the control did not tolerate ethanol concentrations exceeding 10\% (Fig. 1). 


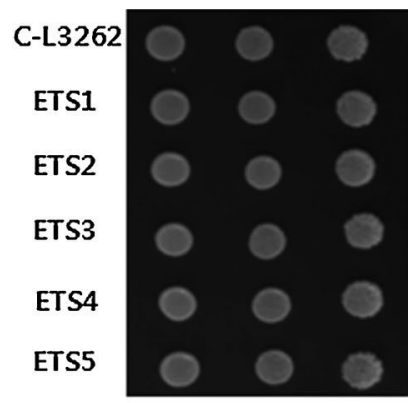

$0 \%$

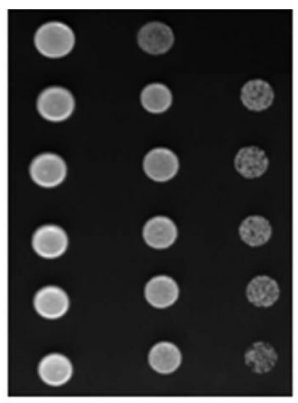

$10 \%$

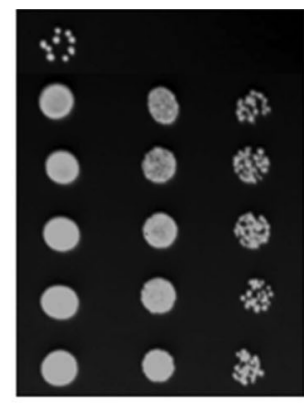

$12.5 \%$

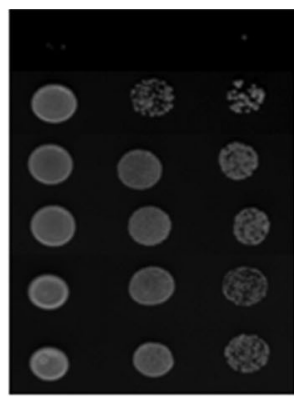

$15 \%$

Figure 1. Enhanced ethanol tolerance of ETS1-5. Cells were grown to an OD600 of 1.0 in the YSCD-Ura or YPD liquid media and tenfold serially diluted. Aliquots (5 $\mu$ l) were spotted onto YSCD-Ura or YPD plates containing appropriate concentrations of ethanol and incubated at $30^{\circ} \mathrm{C}$ for $4-6$ days. Control stain (C-L3262) was constructed by transformation of pSPT15wt into L3262.

To confirm whether the enhanced ethanol tolerance was conferred by the presence of a mutated SPT15, plasmids were recovered from ETS1-5 (pSPT15-M1, -M2, -M3, -M4, and -M5, respectively). Sequencing of each SPT15 allelelocated mutations in the SPT15 ORF: K201N, G216S, and N225Stop in SPT15-M1; L76V and L175S in SPT15-M2; S42N, C78R, S163P, and I212N in SPT15-M3; F10S and M197K in SPT15-M4; K15T, W26C, and G192D in SPT15M5 (Fig. 2). A silent mutation (N225Stop) in SPT15-M1 yielded a truncated version with 16 residues deleted at the Cterminus. No particular point mutation was common, although one or two residues were changed in the repeat element 2 of all alleles. This element contains the domain interacting with Spt3p (amino acid residues 172-179), which has been implicated in the regulation of gene transcription (Alper et al., 2006; Cang et al., 1999). Overall, these data were consistent with the suggestion that the mutation of several subregions of Spt15p confers ethanol tolerance, presumably through the interaction with other components of the transcriptional machinery in addition to Spt3p (Eisenmann et al., 1989).

Each plasmid was re-introduced into L3262 and BY4741 to yield rL-ETS1-5 and rBY-ETS1-5. pRS316-GCYH2gR containing wild-type SPT15 (SPT15wt) was transformed into L3262 and BY4741, yielding control strains C-L3262 and C-BY4741. When spot-assayed on a synthetic medium, rL-ETS1-5 showed the same degree of ethanol tolerance as ETS1-5 did (Fig. 3, top panel). Meanwhile, rBY-ETS1-5 showed tolerance to as high as $17.5 \%$ ethanol (Fig. 3, bottom panel). This was not surprising, since BY4741 originally displayed higher ethanol tolerance than L3262 (data not shown). Thus, the enhanced ethanol tolerance of ETS1-5 was suggested to be the effect of mutated SPT15.

Based on the above spot assay results in which ETS2 and ETS3 were slightly more tolerant than the remainder, these two were chosen for DNA microarray, testing the ability to

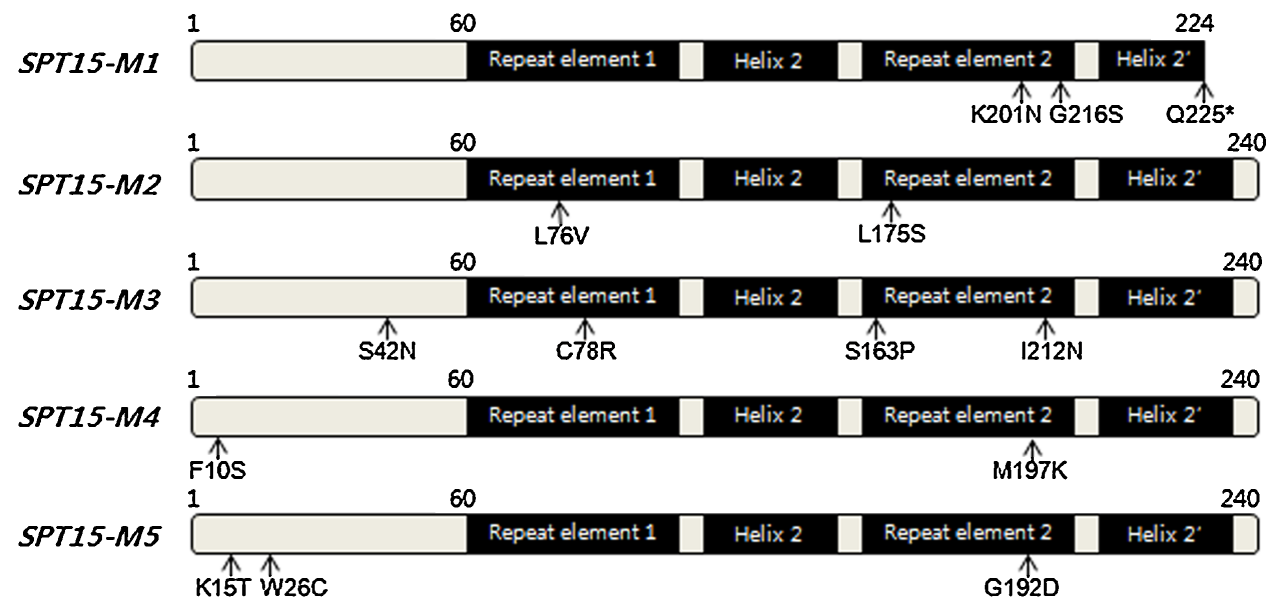

Figure 2. Schematic representation of mutated SPT15 alleles. Plasmids (pSPT15-M1-5) were recovered from ETS1-5 and sequenced. Comparison of the deduced amino acid sequence with SPT15wt locates the position of point mutations (arrows). The map of structural domains is based on the previously published literature (Alper et al., 2006). 


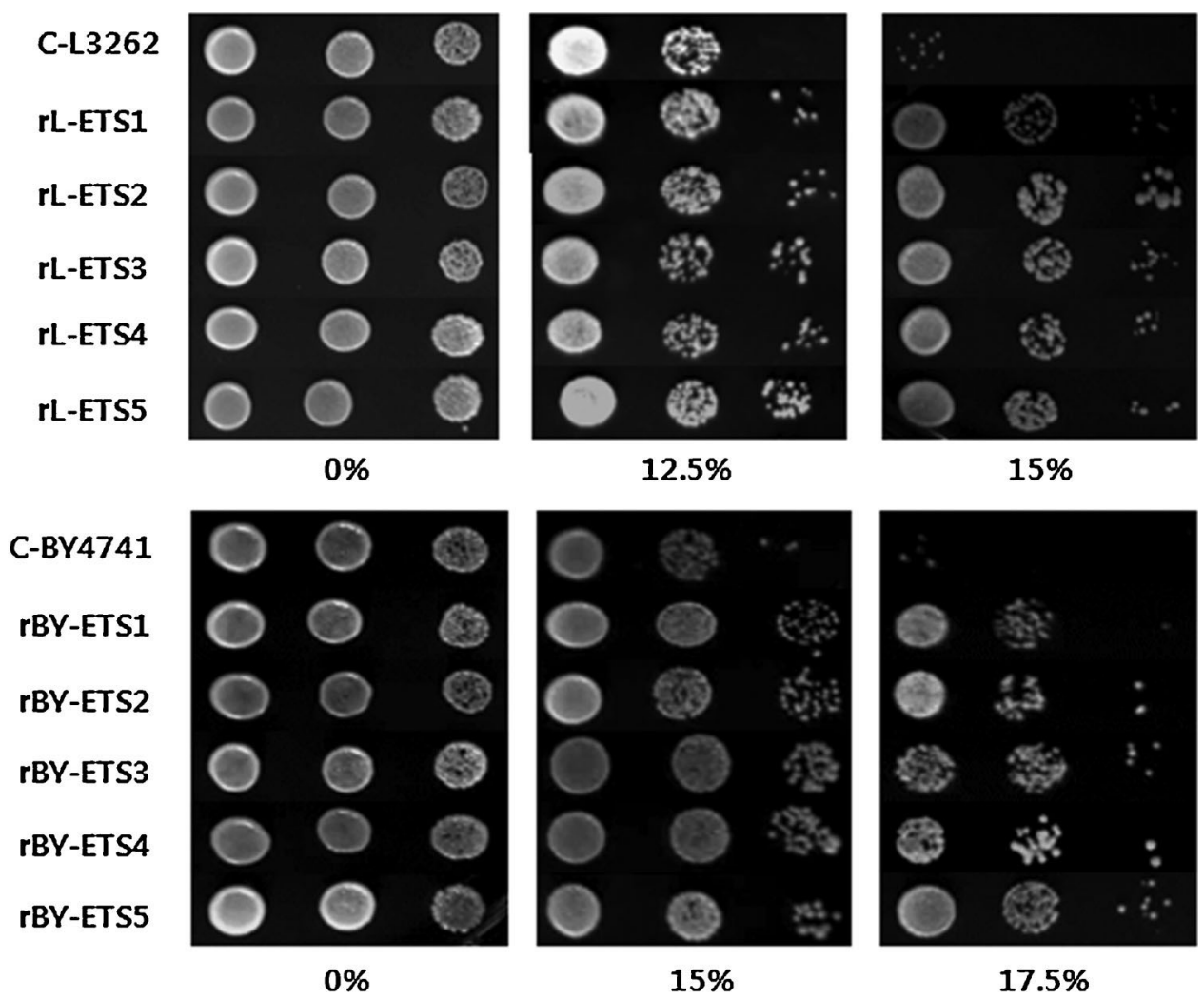

Figure 3. Confirmation of enhanced ethanol tolerance. Plasmids pSPT15-M1-5 were re-transformed into L3262 and BY4741, yielding rL-ETS1-5 and rBY-ETS1-5, respectively. Control stain C-BY4741 was constructed by transformation of pSPT15wt into and BY4741. Spot assay was performed as in Figure 1.

grow on a rich medium, and genome integration of SPT15 alleles.

\section{Transcriptome Profile Analysis of Ethanol-Tolerant Mutant Strains}

We were interested in genes responsible for enhanced ethanol tolerance of ETS2 and ETS3, the expression levels of which were regulated by SPT15 mutations. To obtain this information, DNA microarrays for transcriptional profiling were conducted with total RNAs prepared from control C-L3262, ETS2, and ETS3 cells grown to early-log phase in YSCD-Ura. After performing microarray experiments in duplicate, expression fold changes were averaged. The raw data have been registered at Gene Expression Omnibus under the accession number GSE23965. The level of SPT15 increased eightfold on average in both ETS2 and ETS3 (data not shown). Clustering of genes with fold change higher than two compared to control displayed differential expression patterns between ETS2 and ETS3, reflecting the effect of different mutations of SPT15 on the global transcription (Fig. 4A). To validate the microarray data, the actual expression levels of HSP30, HSP42, and HSP104 were examined by RT-PCR. According to the microarray data,
HSP30, HSP42, and HSP104 were up-regulated by 5.7-, 4.3-, and 1.7-fold in ETS2 and 6.3-, 4.1-, and 1.8-fold in ETS3, respectively. The fold increases of those genes were consistent with the RT-PCR data (Fig. 4B).

In ETS2, 45 and 11 genes were up- and down-regulated, respectively, whereas in ETS3, 79 and 21 genes were up- and down-regulated, respectively (Fig. 4C). Thirty-four upregulated and eight down-regulated genes were shared between ETS2 and ETS3 (Fig. 4C). To gain further information on the transcriptional regulation of commonly up- and down-regulated genes, we examined the presence of putative binding sites for transcription factors presumed to be involved in various stress responses, such as Msn2p/ Msn4p for general stress (Watanabe et al., 2007), Haclp for protein secretion stress (Ogawa and Mori, 2004), Hsflp for heat stress (Yamamoto et al., 2008), and Yaplp for oxidative stress (He and Fassler, 2005). Quite intriguingly, the binding sites for these transcription factors were highly enriched in the upstream regions of commonly up-regulated genes (Table I). Particularly, the binding sites for Msn2p/Msn4p were found in nearly all of commonly up-regulated genes. Meanwhile, the binding sites for Msn4p/Msn2p and Yap1p were found far less frequently, in contrast to similar frequencies for Haclp and Hsflp, in the eight commonly down-regulated genes. The collective data suggests that 
A

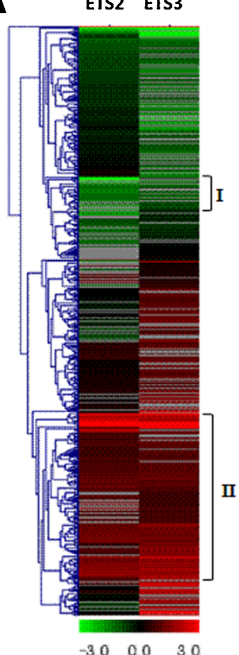

B

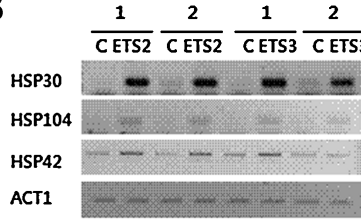

C

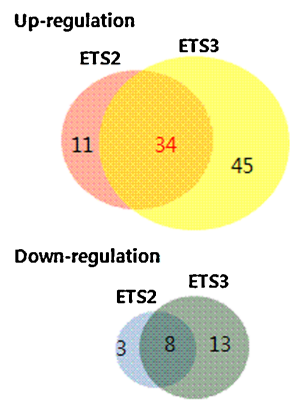

D
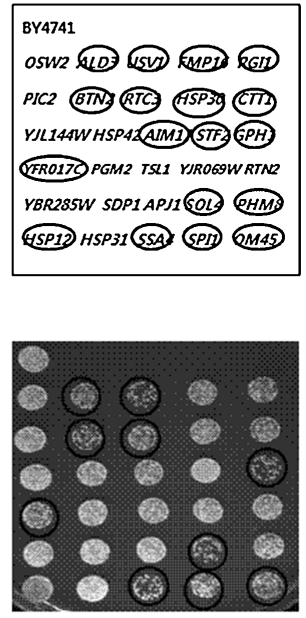

$8 \%$
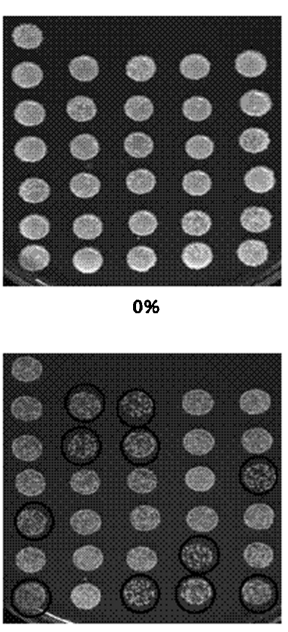

$10 \%$

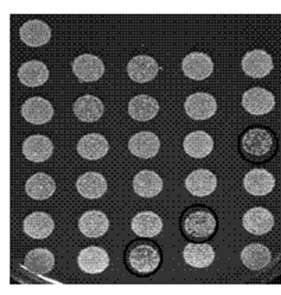

$6 \%$

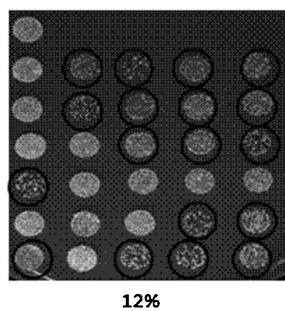

Figure 4. Microarray data analysis of ETS2 and ETS3. Microarray analysis was performed with Poly+ (A). RNAs prepared from C-L3262 (control), ETS2, and ETS3 grown to mid-log phase without ethanol stress challenge. Differentially expressed genes with expression fold change $>2$ were profiled for clustering; I, down-regulated genes, II, upregulated genes. B: Microarray data were validated by semi-quantitative RT-PCR of Hsp30, Hsp42, and Hsp104. Numerals 1 and 2 indicate independent duplicates. C: control (L3262) (C) Venn diagram of up- and down-regulated genes in ETS2 and ETS3. D: Ethanol sensitivity of SGKO mutants. Individual clones corresponding to 30 commonly up-regulated in ETS2 and ETS3 were retrieved from the BY4741 SGKO library. Spot assay was performed as in Figure 1. The parental strain BY4741 was used as a control. Cells were cultured in liquid YPD and spotted on solid YPD containing $0 \%, 6 \%, 8 \%, 10 \%$, and $12 \%$ ethanol, and incubated at $30^{\circ} \mathrm{C}$ for 1-6 days. [Color figure can be seen in the online version of this article, available at http://wileyonlinelibrary.com/bit]

Msn4p/Msn2p and Yap1p may be responsible for the regulation of genes associated with ethanol tolerance.

\section{Effect of Commonly Regulated Genes on Ethanol Tolerance}

Of concern was whether the 34 commonly up-regulated and eight commonly down-regulated genes were a cause or an effect of ethanol tolerance. If the up-regulation of a geneenhanced ethanol tolerance, it would be highly likely that its deletion would render cells sensitive to ethanol. The reverse would be the case for the down-regulated genes. Deletion mutants corresponding to 30 up- and 6 down-regulated genes were retrieved from the BY4741 SGKO collection. Those corresponding to four up-regulated genes (YER053CA, YNR034W-A, YPR145C-A, YBL029C-A) and two downregulated genes (RRN7 and YOR387C) were not available, probably due to their lethality. BY4741 as control and individual deletion mutants grown to an $\mathrm{OD}_{600}$ of 0.5 were diluted tenfold and spotted on solid YPD medium containing several different concentrations of ethanol.

The results for 30 deletion mutants corresponding to commonly up-regulated genes are shown in Figure $4 \mathrm{D}$. Some deletion mutants were sensitive to as low as $6 \%$, far below the concentration that exerts toxic effect to BY4741. It was natural that total number of sensitive mutants increased as the ethanol concentration increased up to $12 \%$. Sensitivity to $6 \%$ ethanol corresponded to deletions in GPH1, SOL4, and SSA4. An additional seven mutants (ALD3, BTN2, SPI1, OM45, RTC3, USV1, and YFR017C) were sensitive to $8 \%$ ethanol. The HSP12 deletion mutant was sensitive to $10 \%$ ethanol. Finally, deletions in HSP30, CTT1, STF2, AIM17, FMP16, RGI1, and PHM8 rendered mutants sensitive to $12 \%$ ethanol. Thus, deletion of 18 out of 30 genes commonly up-regulated in ETS2 and ETS3 conferred ethanol sensitivity. Of these, eight genes (ALD3, BTN2, OM45, RTC3, USV1, YFR017C, FMP16, and PHM8) have never been reported in association with ethanol tolerance or induction upon ethanol shock. Since these genes were identified by combination of DNA microarray and deletion assays, the probability that these genes are involved in the enhanced ethanol tolerance is higher than those identified by either assay. Meanwhile, none of six deletion mutants corresponding to commonly downregulated genes displayed enhanced growth (data not shown), contrary to our expectation that some of them would display higher degree of ethanol tolerance than the control.

\section{Construction of Genome-Integrated Strains}

As shown above, episomal overexpression of mutated SPT15 conferred enhanced ethanol tolerance to cells grown on a synthetic medium. To extend these results to the development of industrial strains, of great concern was whether or not the enhanced ethanol tolerance was sustained in both cells grown on a rich medium and cells with mutated SPT15 alleles integrated into the genome. It has been argued that low leucine supplementation, but not mutated SPT15, led to enhanced ethanol tolerance (Baerends et al., 2009). More 
Table I. Genes commonly regulated in ethanol-tolerant strains ETS2 and ETS3 under no ethanol stress.

\begin{tabular}{|c|c|c|c|c|c|c|c|c|}
\hline \multirow[b]{3}{*}{ Gene } & \multicolumn{4}{|c|}{ Fold change $\left(2^{\mathrm{n}}\right)$} & \multicolumn{4}{|c|}{ Number of binding sites for } \\
\hline & \multicolumn{2}{|c|}{ ETS2 } & \multicolumn{2}{|c|}{ ETS3 } & \multirow[b]{2}{*}{ Msn4p/Msn2p } & \multirow[b]{2}{*}{ Yaplp } & \multirow[b]{2}{*}{ Hsflp } & \multirow[b]{2}{*}{ Haclp } \\
\hline & Exp1 & Exp2 & Exp1 & Exp2 & & & & \\
\hline \multicolumn{9}{|l|}{ Up-regulated } \\
\hline \multicolumn{9}{|c|}{ Stress response and protein folding } \\
\hline APJ1 & 1.4 & 1.1 & 1.3 & 1.3 & 3 & 0 & 2 & 2 \\
\hline$A L D 3$ & 2.5 & 1.8 & 1.6 & 2.1 & 2 & 1 & 0 & 1 \\
\hline$\overline{C T T 1}$ & 1.7 & 1.7 & 1.4 & 1.9 & 4 & 2 & 4 & 1 \\
\hline HSP12 & 1.1 & 1.5 & 1.0 & 1.5 & 7 & 0 & 1 & 4 \\
\hline HSP30 & 2.7 & 2.3 & 3.0 & 2.3 & 0 & 2 & 0 & 0 \\
\hline HSP31 & 1.5 & 1.6 & 4.5 & 1.5 & 1 & 1 & 2 & 0 \\
\hline HSP42 & 2.3 & 1.9 & 1.8 & 2.3 & 3 & 0 & 2 & 1 \\
\hline$S D P 1$ & 2.0 & 1.3 & 1.8 & 1.6 & 3 & 0 & 2 & 0 \\
\hline SSA4 & 1.0 & 1.7 & 1.4 & 1.0 & 3 & 1 & 1 & 1 \\
\hline TSL1 & 1.2 & 1.2 & 1.1 & 1.9 & 7 & 0 & 1 & 2 \\
\hline YJL144W & 3.7 & 2.3 & 3.4 & 2.7 & 1 & 1 & 2 & 2 \\
\hline \multicolumn{9}{|c|}{ Pentose-phosphate pathway } \\
\hline PGM2 & 1.7 & 1.2 & 1.7 & 2.1 & 7 & 1 & 0 & 1 \\
\hline SOL4 & 1.9 & 1.5 & 1.6 & 1.9 & 1 & 0 & 6 & 0 \\
\hline \multicolumn{9}{|l|}{ Cell wall } \\
\hline SPII & 2.0 & 1.3 & 1.8 & 1.6 & 3 & 1 & 2 & 1 \\
\hline OSW2 & 1.2 & 1.0 & 1.9 & 1.4 & 0 & 1 & 2 & 1 \\
\hline \multicolumn{9}{|l|}{ Transport } \\
\hline PIC2 & 1.5 & 1.1 & 1.4 & 1.1 & 1 & 0 & 2 & 0 \\
\hline BTN2 & 2.9 & 3.1 & 2.9 & 3.5 & 2 & 0 & 1 & 0 \\
\hline \multicolumn{9}{|c|}{ Metabolism of energy reserves } \\
\hline GPH1 & 1.1 & 1.3 & 1.2 & 2.1 & 3 & 1 & 0 & 1 \\
\hline Energy generatic & & & & & & & & \\
\hline$S T F 2$ & 1.7 & 1.2 & 1.8 & 1.4 & 2 & 1 & 1 & 2 \\
\hline Unclassified pro & & & & & & & & \\
\hline AIM17 & 2.4 & 1.0 & 1.8 & 1.9 & 3 & 0 & 2 & 2 \\
\hline FMP16 & 1.2 & 1.3 & 1.0 & 1.6 & 1 & 1 & 0 & 0 \\
\hline$O M 45$ & 1.4 & 1.0 & 1.0 & 1.5 & 3 & 1 & 4 & 3 \\
\hline$\overline{P H M 8}$ & 1.5 & 1.6 & 1.6 & 1.0 & 4 & 0 & 2 & 0 \\
\hline$\overline{\mathrm{RTC3}}$ & 2.4 & 2.1 & 2.1 & 2.6 & 4 & 1 & 0 & 0 \\
\hline$\overline{R T N 2}$ & 3.4 & 1.7 & 1.6 & 1.4 & 1 & 0 & 2 & 0 \\
\hline USVI & 2.4 & 1.0 & 1.8 & 1.7 & 6 & 1 & 0 & 0 \\
\hline$\overline{R G I I}$ & 3.3 & 1.1 & 3.1 & 2.2 & 4 & 1 & 4 & 3 \\
\hline$Y B L 029 C-A$ & 1.3 & 1.0 & 1.3 & 1.1 & 4 & 1 & 0 & 0 \\
\hline YBR285W & 1.7 & 1.2 & 1.6 & 1.3 & 2 & 1 & 2 & 1 \\
\hline YER053C-A & 1.3 & 1.1 & 1.3 & 1.0 & 2 & 0 & 0 & 0 \\
\hline YFR017C & 3.1 & 1.1 & 2.6 & 3.6 & 2 & 0 & 0 & 1 \\
\hline$\overline{Y J R 096 W}$ & 1.4 & 1.3 & 1.7 & 1.6 & 1 & 1 & 2 & 1 \\
\hline YNR034W-A & 3.4 & 2.5 & 3.3 & 3.1 & 5 & 1 & 0 & 0 \\
\hline YPR145C-A & 1.3 & 1.0 & 1.6 & 1.0 & 0 & 0 & 0 & 0 \\
\hline Down-regulated & & & & & & & & \\
\hline Budding cell po & d filam & nation & & & & & & \\
\hline$R A X 2$ & -1.3 & -1.0 & -1.0 & -1.5 & 0 & 1 & 2 & 0 \\
\hline C-Compound a & ohydra & olism & & & & & & \\
\hline$B S C 1$ & -2.1 & -2.1 & -1.5 & -2.2 & 3 & 1 & 1 & 0 \\
\hline Mating (fertiliza & & & & & & & & \\
\hline PRM7 & -1.0 & -1.0 & -1.3 & -1.2 & 0 & 0 & 0 & 2 \\
\hline Protein targeting & and $\mathrm{tr}$ & ion & & & & & & \\
\hline VTS1 & -1.5 & -1.1 & -1.1 & -1.1 & 2 & 0 & 2 & 0 \\
\hline rRNA synthesis & & & & & & & & \\
\hline RRN7 & -1.7 & -1.3 & -4.0 & -1.2 & 2 & 0 & 2 & 2 \\
\hline Unclassified & & & & & & & & \\
\hline$V E L 1$ & -2.6 & -1.0 & -1.2 & -5.1 & 0 & 0 & 2 & 0 \\
\hline YGR035C & -1.8 & -1.1 & -1.1 & -1.7 & 0 & 0 & 2 & 1 \\
\hline YOR387C & -2.4 & -1.0 & -1.1 & -4.5 & 0 & 0 & 2 & 1 \\
\hline
\end{tabular}

Genes showing more than twofold change are listed. Genes whose deletion renders cells sensitive to ethanol are in bold (Fig. 4). Of these, genes that have never been reported are underlined. 
significantly, ethanol tolerance was abolished when cells were cultured in the YPD-rich medium, which is not optional for industrial applications. Since ETS2 and ETS3 are leucine auxotrophs, the enhanced ethanol tolerance of these strains might not be due to mutations in SPT15. However, ETS2 and ETS3 sustained ethanol tolerance on YPD as shown in Figure 5A.

The cell-cell heterogeneity in expression is one of issues encountered when the information obtained from episomal overexpression in laboratory strains is scaled up to industrial applications. Heterogeneity is caused by the inability to control copy number in spite of the continual presence of selection pressure, which clearly is not optional for yeast culture on an industrial scale. Accordingly, stable expression and maintenance of the gene in the absence of selective pressure (i.e., integration into the chromosome) is frequently desirable. Here, we constructed strains in which SPT15-M2 and -M3 were integrated into the genome of L3262; the corresponding constructs were named iETS2 and
iETS3, respectively. The control strain iL3262 were created with a plasmid containing SPT15wt. Figure 5B shows that iETS2 and iETS3 displayed enhanced ethanol tolerance on YPD.

To confirm the spot assay results, the susceptibility to $12.5 \%$ and $15 \%$ ethanol was examined for iETS2 and iETS3 (Fig. 5C). At $12.5 \%$ ethanol, the timepoint showing 50\% viability $\left(T_{50}\right)$ was $4.5 \mathrm{~h}$ for iETS2 and iETS3, in contrast to $3.5 \mathrm{~h}$ for the control. A sharper contrast was observed at $15 \%$ ethanol, with a $T_{50}$ of $100 \mathrm{~min}$ for both iETS2 and iETS3, and $40 \mathrm{~min}$ for control. Thus, we obtained two integrated strains with enhanced ethanol tolerance on a rich medium.

\section{The Effect of Mutated SPT15s on Ethanol Production}

Our next concern was to investigate the relevance of enhanced ethanol resistance of iETS2 and iETS3 (compared to control iL3263) to improvement of ethanol production
A L3262 ETS2 ETS3

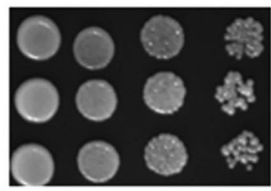

$0 \%$

B

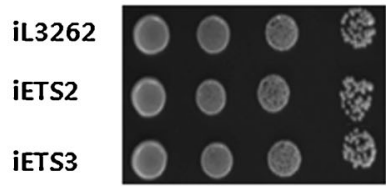

0\%

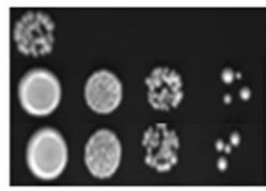

$12.5 \%$

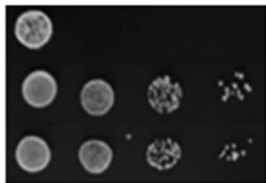

$12.5 \%$

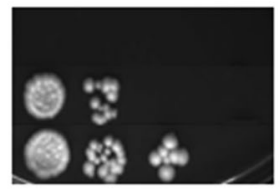

$15 \%$

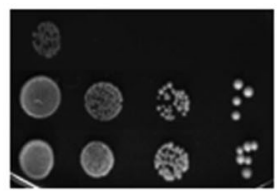

$15 \%$
C

$12.5 \%$ ethanol

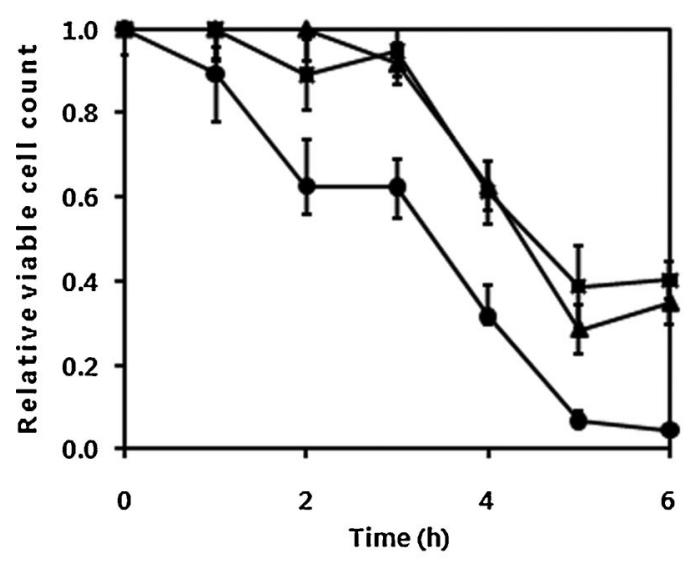

$15 \%$ ethanol

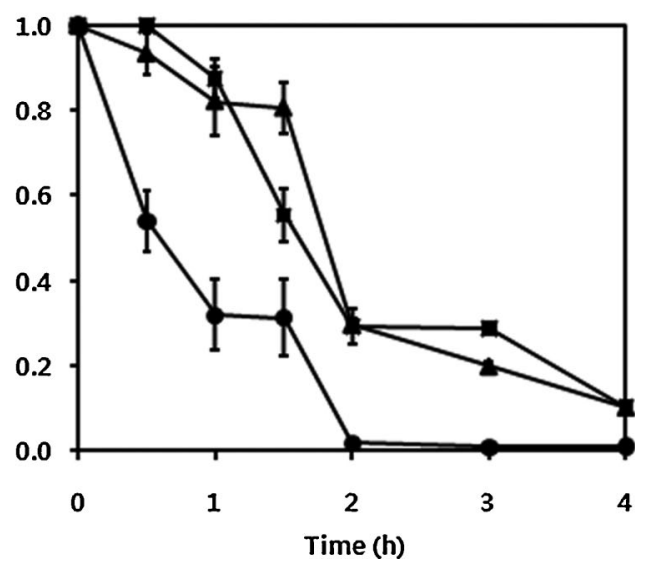

Figure 5. Ethanol tolerance of episomal and integrated ETS2 and ETS3 on YPD. A: Spot assay of ETS2 and ETS3 on the YPD plate. B: The parental plasmid and plasmids recovered from ETS2 and ETS3 were integrated into the genome of L3262, yielding iL3262, iETS2, and iETS3, respectively. The spot assay was performed on the YSCD-Ura (top panel) and YPD plates (bottom panel). C: Ethanol susceptibility of iETS3 and iETS3. Following ethanol shock for the indicated times, iL3262 ( $)$ ), iETS2 ( $\mathbf{\square}$ ), and iETS3 ( $\mathbf{\Delta}$ ) were grown on the YSCD-Ura plate in the presence of $12.5 \%$ and $15 \%$ ethanol for $4-6 \mathrm{~h}$. Relative viability was expressed as percentage after counting the number of colony. Experiments were done in triplicate. 
under a certain condition. During the 24-h-long ethanolic fermentations performed in YPD20 media as described in Materials and Methods section, the cell growth and ethanol productivity of iL3262 (control), iETS2, and iETS3 were examined (Fig. 6). Three strains grew at the same rate until $4 \mathrm{~h}$ timepoint (Fig. 6A), at which the ethanol concentration in the medium was below $1 \%$ (Fig. 6B), and then iETS2 and iETS3 grew faster than control, indicating that such low concentration of ethanol started to affect the cell growth. The growth of control seemed to cease at $16 \mathrm{~h}$ timepoint
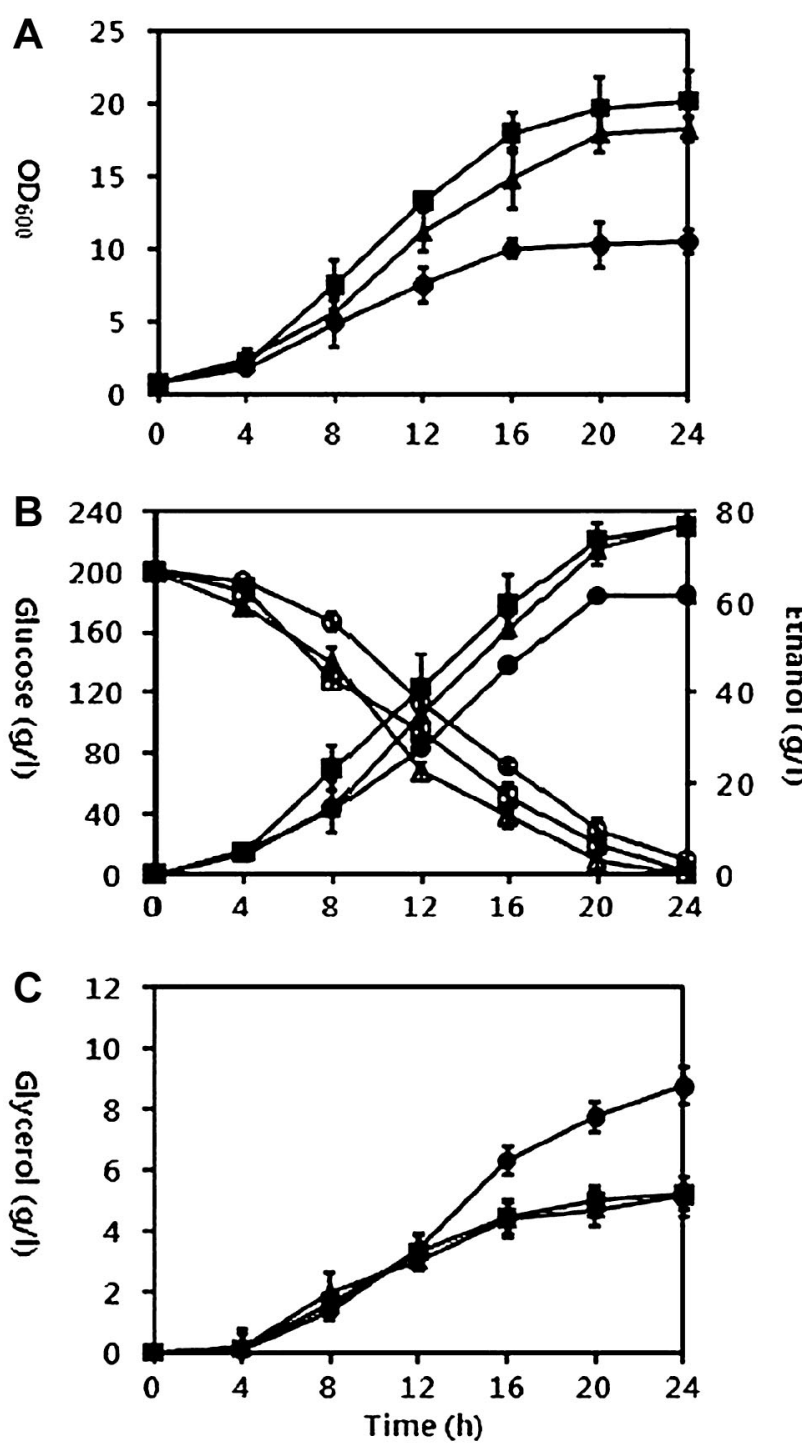

Figure 6. Fermentation kinetics of engineered strains. Overnight yeast cells of control iL3262 (circle) and two ethanol-tolerant strains, iETS2 (square) and iETS3 (triangle), were harvested and transferred to a $1-\mathrm{L}$ bioreactor containing $500 \mathrm{~mL}$ of YPD20 (2\% peptone, $1 \%$ yeast extract, $0.02 \%$ (NH4) 2S04, and $20 \%$ glucose) with the initial cell density adjusted to $0 D 600$ of 1.0. Ethanolic fermentations were performed as described in Materials and Methods section. Samples were taken at $4 \mathrm{~h}$ intervals to measure the optical density for cell growth $(\mathbf{A})$ and to determine the concentrations of glucose (B, open), ethanol (B, closed), and glycerol (C) by using HPLC. Experiments were done in triplicate. where the ethanol concentration was 5.8\%, while iETS2 and iETS3 continued to grow for $4 \mathrm{~h}$ or longer, yielding significant differences in $\mathrm{OD}_{600}$. At $24 \mathrm{~h}$ timepoint where glucose added was almost completely consumed, the final amounts of ethanol were $77 \mathrm{~g} / \mathrm{L}$ for both iETS2 and iETS3 and $61.5 \mathrm{~g} / \mathrm{L}$ for control. The ethanol yield for iETS2 and iETS3 $(0.39 \mathrm{~g} / \mathrm{g})$ was increased by $26 \%$ from $0.31 \mathrm{~g} / \mathrm{g}$ for control. The low ethanol yield by control might be due to the strain specificity. The ethanol productivity of iETS2 and iETS3 was substantially enhanced by $23 \%$ (from 2.6 to $3.2 \mathrm{~g} /$ $\mathrm{L} / \mathrm{h}$ ), although the specific ethanol productivity ( $\mathrm{g}$ ethanol/g cell/h) of the control strain was higher than iETS2 and iETS3 due to much less growth of control $\left(\sim 10 \mathrm{OD}_{600}\right)$ compared with iETS2 and iETS3 $\left(\sim 20 \mathrm{OD}_{600}\right.$, Fig. $\left.6 \mathrm{~A}\right)$. The less ethanol production in spite of higher specific ethanol productivity of control was presumed to result from the cease of cell growth at $16 \mathrm{~h}$ timepoint, where the ethanol concentration was approximately $5.8 \%$ (Fig. 6B). The less cell mass and lower ethanol yield by control during fermentation seem to be attributed at least in part to relatively higher conversion of glucose to glycerol starting from $12 \mathrm{~h}$ time point at which the ethanol concentration was approximately $3.5 \%$ (Fig. $6 \mathrm{C}$ ). These data together suggest that the ethanol concentration of as low as $1 \%$ negatively affects the cell growth and $3.5 \%$ ethanol is enough to change the glucose metabolism in iL3262, and further that iETS2 and iETS3 overcome these adversities in favor of efficient ethanol production by the enhanced ethanol tolerance conferred by mutated SPT15 alleles.

\section{Discussion}

The development of ethanol-tolerant $S$. cerevisiae strains is of great economic value for brewing and beverage industries, and the manufacture of bioethanol. The organism-based traditional approaches, such as evolutionary adaptation, random chemical mutagenesis, and gene shuffling, have shed little light on the understanding of the molecular mechanisms of ethanol tolerance. Over the past decade, DNA microarray and SGKO library screening have been extensively exploited to identify genes that are presumed to be associated with ethanol tolerance. Still, the mechanism of ethanol tolerance remains largely unknown. In the meantime, several ethanol strains have been developed by gene manipulations, including a few successful cases based on the data from DNA microarray (Hirasawa et al., 2007) and SGKO library screening (Yazawa et al., 2007), and overexpression of sonicated genomic fragments (Hong et al., 2010). Several strategies identified 18 autologous and two heterologous genes whose deletion, disruption, depletion, or overexpression to the parental cells (Supplementary Table 2). In the present study, 18 genes whose up-regulation is very likely to confer ethanol tolerance were identified by the combined use of DNA microarray and SGKO library from two ETSs constructed by overexpression of two different SPT15 mutant alleles SPT15-M2 and -M3 (Fig. 4A). 
Intriguingly, none of the autologous genes conferring tolerance by overexpression overlapped with the 18 genes, suggesting that the ethanol tolerance associated with those genes is independent of that resulting from the overexpression of both SPT15-M2 and -M3.

Prior to the advent of the DNA microarray, the DNA filter array analysis identified six genes (GPD1, CTT1, HSP12, SpI1, TPS1, and TPS2) up-regulated in an ethanol-tolerant sake strain compared to the parental strain cultivated free from ethanol stress (Ogawa et al., 2000). Since then, DNA microarray, which has identified a large number of candidate genes associated with ethanol tolerance by comparing the expression profiles of controls (C) and the same cells challenged with ethanol (e-C) under varied conditions. A recent elaborate study (Ma and Liu, 2010a,b) compared the quantitative transcription dynamics of 175 selected genes presumed to be related to ethanol tolerance between $\mathrm{C}$ and $\mathrm{e}-\mathrm{C}$, between $\mathrm{C}$ and $\mathrm{T}_{\mathrm{i}}$ (isogenic ETS), and between $\mathrm{C}$ and $\mathrm{e}-\mathrm{T}_{\mathrm{i}}$ ( $\mathrm{Ti}$ challenged with ethanol), and revealed significant variation in gene activities depending on the comparison set and sampling timepoint. The results of these and other studies and the present study are summarized in Table II. Ma and Liu (2010b) also showed that all 34 genes of e-C overlapped with e- $\mathrm{T}_{\mathrm{i}}$ and six genes (HSP31, IRC15, ADH1, ADH2, ADH3, and ADH7) were common between $\mathrm{T}_{\mathrm{i}}$ and $\mathrm{e}-\mathrm{T}_{\mathrm{i}}$. These data imply that ethanol challenge induces a similar set of genes regardless whether a strain is ethanol tolerant or not, and that the expression profile of an unchallenged ETS is considerably different from that of challenged ETS. We propose that ethanol tolerance should be dealt with separately from ethanol induction. In fact, PUT1, ATH1, and NTH1, which confer ethanol tolerance when deleted or disrupted (Supplementary Table 2), were up-regulated upon ethanol induction (Ma and Liu, 2010b): sixfold in e-C and fivefold in e- $\mathrm{T}_{\mathrm{i}}$ for PUT1; 2.2-fold in e- $\mathrm{T}_{\mathrm{i}}$ for ATH1; 2.0-fold in e-C and 2.7-fold in $\mathrm{e}-\mathrm{T}_{\mathrm{i}}$ for $\mathrm{NTH1}$. Among the genes constitutively up-regulated in four ETSs (SR4-3, Y50316, ETS2, and ETS3), only three genes (CTT1, HSP12, and SPI1) were common between SR4-3, ETS2, and ETS3. Considering the methods employed for developing the strains used for each assay (i.e., breeding for SR4-3, adaptation evolutionary engineering for Y50316, and gTME for ETS2 and ETS3), ethanol tolerance may be achieved by several independent routes in $S$. cerevisiae.

When the construction of a strain with enhanced high glucose and ethanol tolerance by exploiting gTME was reported (Alper et al., 2006), this technique seemed to be a promising approach to directly reveal multiple stress tolerance. Such genetic traits were claimed to be conferred by a specific SPT15 mutant allele (SPT15-300), which resulted from reprogramming of the global gene expression. However, no follow-up studies have been reported, and neither ethanol nor high glucose tolerance trait was observed when cells containing the SPT15-300 allele were grown on rich media (Baerends et al., 2009). In the present study, five ethanol-tolerant $S$. cerevisiae strains were constructed by exploiting gTME. The ethanol tolerance phenotype was sustained when cells were grown on YPD agar (Fig. 5A). This discrepancy may be due to differential expression of a set of

Table II. Comparison sets and strategies used to identify ethanol tolerance-related genes by comparing expression profiles.

\begin{tabular}{|c|c|c|c|c|c|}
\hline \multicolumn{2}{|c|}{ Set } & \multirow[b]{2}{*}{ Strategy used } & \multicolumn{2}{|c|}{$\begin{array}{c}\text { Number of } \\
\text { regulated genes }\end{array}$} & \multirow[b]{2}{*}{ Source } \\
\hline Control & Counterpart & & Up & Down & \\
\hline $\mathrm{C}$ & $e-C$ & & & & \\
\hline $\mathrm{NS}^{\mathrm{b}}$ & $\mathrm{e}-\mathrm{NS}{ }^{\mathrm{b}}$ & Microarray & $200-400$ & NA & $\begin{array}{l}\text { Alexandre et al. (2001); Chandler et al. (2004); } \\
\text { Dinh et al. (2009); Hirasawa et al. (2007); } \\
\text { Marks et al. (2008); Rossignol et al. (2003); } \\
\text { Varela et al. (2005); Wu et al. (2006) }\end{array}$ \\
\hline Y50049 & e-Y50049 & qRT-PCR ${ }^{\mathrm{c}}$ & $34^{\mathrm{d}}$ & $50^{\mathrm{d}}$ & Ma and Liu (2010b) \\
\hline $\mathrm{C}$ & $\mathrm{T}_{\mathrm{i}}$ & & & & \\
\hline K701 & SR4-3 & Filter screening & 6 & NA & Ogawa et al. (2000) \\
\hline Y50049 & Y50316 & qRT-PCR ${ }^{c}$ & $12^{\mathrm{d}}$ & $5^{\mathrm{d}}$ & Ma and Liu (2010b) \\
\hline L3262 & ETS2 & Microarray & $45\left(34^{\mathrm{e}}\right)$ & $11\left(8^{\mathrm{e}}\right)$ & This study \\
\hline L3262 & ETS3 & Microarray & $79\left(34^{\mathrm{e}}\right)$ & $21\left(8^{\mathrm{e}}\right)$ & This study \\
\hline $\mathrm{C}$ & $e-T_{i}$ & & & & \\
\hline Y50049 & e-Y50316 & qRT-PCR ${ }^{c}$ & $41^{\mathrm{d}}$ & $16^{\mathrm{d}}$ & Ma and Liu (2010b) \\
\hline $\mathrm{e}-\mathrm{C}$ & $e-T_{u}$ & & & & \\
\hline X2180-1A & e-K9 & Microarray & $283^{\mathrm{f}}$ & $167^{\mathrm{f}}$ & Shobayashi et al. (2007) \\
\hline
\end{tabular}

The prefix ' $\mathrm{e}$ ' represents ethanol challenge. The subscripts indicate that the counterpart is isogenic (i) or unrelated (u) to control. Strains used are in parentheses. C, control strain; T, ethanol-tolerant strain; NA, not available.

${ }^{\mathrm{a}}$ Based on twofold change.

${ }^{\mathrm{b}}$ Not specified here due to the variety.

${ }^{c}$ Performed for 175 genes selected from previous studies for an ethanol-tolerant yeast and its parental strain.

dShowing twofold change at any timepoint during 48-h-long ethanol challenge.

${ }^{\mathrm{e}}$ Common between ETS2 and ETS3.

${ }_{\mathrm{f}}^{\mathrm{f}}$ Regulated under either shaking or static condition. 
genes induced by different SPT15 alleles (SPT15-300 and SPT15-M2 or -M3) as shown in the DNA microarray analysis. Most notably, genes involved in the amino acid metabolism including leucine, the elevation of which has been suspected to closely associated with ethanol tolerance conferred by SPT15-300 (Baerends et al., 2009), were not upregulated by SPT15-M2 or -M3 (Table I). The differential expression cannot be simply explained by the difference in location of point mutations: three of SPT15-300 (F177S, $\mathrm{Y} 195 \mathrm{H}$, and K218R) clustered in the repeat element 2 (Alper et al., 2006), whereas those of SPT15-M1-5 scattered on the SPT15 ORF without overlapping with each other, including the non-conserved $\mathrm{N}$-terminal region of 60 amino acids (Fig. 2). Even a truncated SPT15 (SPT15-M1) was formed. However, it should be noted that an amino acid change(s) was commonly found in the repeat element 2 of SPT15-M15 , suggesting that this domain might be related with ethanol tolerance observed in this study. It would be interesting to determine which amino acids are responsible for ethanol tolerance by reverting mutations to their wild types or shuffling mutations between SPT15-M1-5.

We also showed that the enhanced ethanol tolerance conferred by gTME through mutated SPT15 alleles resulted in $25 \%$ increase of ethanol production (Fig. 6). Previously, the effect of enhanced ethanol tolerance generated in various ways such as genomic shotgun (Hong et al., 2010), SGKO screening (Teixeira et al., 2009), genome shuffling (Hou, 2009) and transcription factor overexpression (Hou et al., 2009), was examined by measuring the highest ethanol titer from batch cultures. In these studies, however, the ethanol production was increased by slightly more than $10 \%$ compared to the control strains. The contrasting increase in ethanol production between the previous and present studies may be due to different fermentation conditions: most probably, flask culture versus bioreactor culture. Although it is difficult to compare the efficiency of several technologies mentioned above, gTME seems to be an efficient tool to create strains with enhanced ethanol tolerance. In addition, the SPT15 mutation library constructed in this study may be further used for screening strains with enhanced tolerance to other stresses such as heat, fermentation inhibitors, osmotic pressure, and so on.

We are grateful to Dr. Won-Kee Hur (Seoul National University, Korea) for providing S. cerevisiae BY4741 deletion mutant library and to Drs. J. B. Park (Ewha Womans University, Korea) and Y. C. Park (Kukmin University, Korea) for helpful comments especially in fermentation. This research was supported by Pioneer Research Center Program through the National Research Foundation of Korea funded by Ministry of Education, Science and Technology (No. 20090081512 and No. 2007-2005047).

\section{References}

Alexandre H, Ansanay-Galeote V, Dequin S, Blondin S. 2001. Global gene expression during short-term ethanol stress in Saccharomyces cerevisiae. FEBS Lett 498:98-103.

Alper H, Moxley J, Nevoigt E, Fink GR, Stephanopoulos G. 2006. Engineering yeast transcription machinery for improved ethanol tolerance and production. Science 314:1565-1568.
Baerends RJ, Qiu JL, Rasmussen S, Nielsen HB, Brandt A. 2009. Impaired uptake and/or utilization of leucine by Saccharomyces cerevisiae is suppressed by the SPT 15-300 allele of the TATA-binding protein gene. Appl Environ Microbiol 75:6055-6061.

Cang Y, Auble DT, Prelich G. 1999. A new regulatory domain on the TATAbinding protein. EMBO J 18:6662-6671.

Chandler M, Stanley GA, Rogers P, Chambers P. 2004. A genomic approach to defining the ethanol stress response in the yeast Saccharomyces cerevisiae. Ann Microbiol 54:427-454.

Ding J, Huang X, Zhang L, Zhao N, Yang D, Zhang K. 2009. Tolerance and stress response to ethanol in the yeast Saccharomyces cerevisiae. Appl Microbiol Biotechnol 85:253-263.

Dinh TN, Nagahisa K, Yoshikawa K, Hirasawa T, Furusawa C, Shimizu H. 2009. Analysis of adaptation to high ethanol concentration in Saccharomyces cerevisiae using DNA microarray. Bioprocess Biosyst Eng 32:681-688.

Eisenmann DM, Dollard C, Winston F. 1989. SPT15, the gene encoding the yeast TATA binding factor TFIID, is required for normal transcription initiation in vivo. Cell 58:1183-1191.

Fujita K, Matsuyama A, Kobayashi Y, Iwahashi H. 2006. The genomewide screening of yeast deletion mutants to identify the genes required for tolerance to ethanol and other alcohols. FEMS Yeast Res 6:744-750.

Gibson BR, Lawrence SJ, Leclaire JP, Powell CD, Smart KA. 2007. Yeast responses to stresses associated with industrial brewery handling. FEMS Microbiol Rev 31:535-569.

Gietz RD, Woods RA. 2002. Transformation of yeast by lithium acetate/ single-stranded carrier DNA/polyethylene glycol method. Methods Enzymol 350:87-96.

He XJ, Fassler JS. 2005. Identification of novel Yaplp and Skn7p binding sites involved in the oxidative stress response of Saccharomyces cerevisiae. Mol Microbiol 58:1454-1467.

Hirasawa T, Yoshikawa K, Nakakura Y, Nagahisa K, Furusawa C, Katakura Y, Shimizu H, Shioya S. 2007. Identification of target genes conferring ethanol stress tolerance to Saccharomyces cerevisiae based on DNA microarray data analysis. J Biotechnol 131:34-44.

Hong ME, Lee KS, Yu BJ, Sung YJ, Park SM, Koo HM, Kweon DH, Park JC, Jin YS. 2010. Identification of gene targets eliciting improved alcohol tolerance in Saccharomyces cerevisiae through inverse metabolic engineering. J Biotechnol 149:52-59.

Hou L. 2009. Novel methods of genome shuffling in Saccharomyces cerevisiae. Biotechnol Lett 31:671-677.

Hou L, Cao X, Wang C, Lu M. 2009. Effect of overexpression of transcription factors on the fermentation properties of Saccharomyces cerevisiae industrial strains. Lett Appl Microbiol 49:14-19.

Kubota S, Takeo I, Kume K, Kanai M, Shitamukai A, Mizunuma M, Miyakawa T, Shimoi H, Iefuji H, Hirata D. 2004. Effect of ethanol on cell growth of budding yeast: Genes that are important for cell growth in the presence of ethanol. Biosci Biotechnol Biochem 68:968972.

Ma M, Liu ZL. 2010a. Mechanisms of ethanol tolerance in Saccharomyces cerevisiae. Appl Microbiol Biotechnol 87:829-845.

Ma M, Liu ZL. 2010b. Quantitative transcription dynamic analysis reveals candidate genes and key regulators for ethanol tolerance in Saccharomyces cerevisiae. BMC Microbiol 10:169-188.

Marks VD, Ho Sui SJ, Erasmus D, van der Merwe GK, Brumm J, Wasserman WW, Bryan J, van Vuuren HJJ. 2008. Dynamics of the yeast transcriptome during wine fermentation reveals a novel stress response. FEMS Yeast Res 8:35-52.

Ogawa N, Mori K. 2004. Autoregulation of the HAC1 gene is required for sustained activation of the yeast unfolded protein response. Genes Cells 9:95-104.

Ogawa Y, Nitta A, Uchiyama H, Imamura T, Shiomoi H, Ito K. 2000. Tolerance mechanism of the ethanol-tolerant mutant of sake yeast. J Biosci Bioeng 90:313-320.

Oh KS, Kwon O, Oh YW, Sohn MJ, Jung S, Kim YK, Kim MG, Rhee SK, Gellissen G, Kang HA. 2004. Fabrication of a partial genome microarray of the methylotrophic yeast Hansenula polymorpha: Optimization and 
evaluation for transcript profiling. J Microbiol Biotechnol 14:12391248.

Park JN, Sohn MJ, Oh DB, Kwon O, Rhee SK, Hur CG, Lee SY, Gellissen G, Kang HA. 2007. Identification of the cadmium-inducible Hansenula polymorpha SEO1 gene promoter by transcriptome analysis and its application to whole-cell heavy-metal detection systems. Appl Environ Microbiol 73:5990-6000.

Pieler R, Sanchez-Cabo F, Hackl H, Thallinger GG, Trajanoski Z. 2004. ArrayNorm: Comprehensive normalization and analysis of microarray data. Bioinformatics 20:1971-1973.

Rossignol T, Dulau L, Julien A, Blondin B. 2003. Genome-wide monitoring of wine yeast gene expression during alcoholic fermentation. Yeast 20:1369-1385.

Sambrook J, Russell D. 2001. Molecular cloning: A laboratory manual, 3rd edition. New York: Cold Spring Harbor Laboratory Press.

Shobayashi M, Ukena E, Fujii T, Iefuji H. 2007. Genome-wide expression profiles of sake brewing yeast under shaking and static conditions. Biosci Biotechnol Biochem 71:323-335.

Teixeira MC, Raposo LR, Mira NP, Lourenco AB, Sa-Correia I. 2009. Genome-wide identification of Saccharomyces cerevisiae genes required for maximal tolerance to ethanol. Appl Environ Microbiol 75:5761-5772.

van Voorst F, Houghton-Larsen J, Jonson L, Kielland-Brandt MC, Brandt A. 2006. Genome-wide identification of genes required for growth of Saccharomyces cerevisiae under ethanol stress. Yeast 23:351-359.
Varela CJ, Cardenas J, Melo F, Agosin E. 2005. Quantitative analysis of wine yeast gene expression profiles under winemaking conditions. Yeast 22:369-383.

Watanabe M, Tamura K, Magbanua JP, Takano K, Kitamoto K, Kitagaki H, Akao T, Shimoi H. 2007. Elevated expression of genes under the control of stress response element (STRE) and Msn2p in an ethanol-tolerance sake yeast Kyokai no. 11. J Biosci Bioeng 104: 163-170.

Wu H, Zheng X, Araki Y, Sahara H, Takagi H, Shimoi H. 2006. Global gene expression analysis of yeast cells during sake brewing. Appl Environ Microbiol 72:7353-7358.

Yamamoto N, Maeda Y, Ikeda A, Sakurai H. 2008. Regulation of thermotolerance by stress-induced transcription factors in Saccharomyces cerevisiae. Eukaryot Cell 7:783-790.

Yazawa H, Iwahashi H, Uemura H. 2007. Disruption of URA7 and GAL6 improves the ethanol tolerance and fermentation capacity of Saccharomyces cerevisiae. Yeast 24:551-560.

Yoshikawa K, Tanaka T, Furusawa C, Nagahisa K, Hirasawa T, Shimizu H. 2009. Comprehensive phenotypic analysis for identification of genes affecting growth under ethanol stress in Saccharomyces cerevisiae. FEMS Yeast Res 9:32-44.

Zhao XQ, Bai FW. 2009. Mechanisms of yeast stress tolerance and its manipulation for efficient fuel ethanol production. J Biotechnol 144:23-30. 